\title{
Detecting protein-induced folding of the U4 snRNA kink-turn by single-molecule multiparameter FRET measurements
}

\author{
ANNA K. WOŹNIAK, ${ }^{1}$ STEPHANIE NOTTROTT, ${ }^{2,4}$ EVA KÜHN-HÖLSKEN, ${ }^{2}$ \\ GUNNAR F. SCHRÖDER, ${ }^{3}$ HELMUT GRUBMÜLLER, ${ }^{3}$ REINHARD LÜHRMANN, ${ }^{2}$ \\ CLAUS A.M. SEIDEL, ${ }^{1}$ and FILIPP OESTERHELT ${ }^{\mathbf{1}}$ \\ ${ }^{1}$ Heinrich-Heine-Universität Düsseldorf, Institut für molekulare Physikalische Chemie, Universitätsstraße 1, \\ 40225 Düsseldorf, Germany \\ ${ }^{2}$ Max-Planck-Institut für biophysikalische Chemie, Zelluläre Biochemie, Am Faßberg 11, D-37077 Göttingen, Germany \\ ${ }^{3}$ Max-Planck-Institut für biophysikalische Chemie, Theoretische und computergestützte Biophysik, Am Faßberg 11, D-37077 \\ Göttingen, Germany
}

\begin{abstract}
The kink-turn (k-turn), a new RNA structural motif found in the spliceosome and the ribosome, serves as a specific protein recognition element and as a structural building block. While the structure of the spliceosomal U4 snRNA k-turn/15.5K complex is known from a crystal structure, it is unclear whether the k-turn also exists in this folded conformation in the free U4 snRNA. Thus, we investigated the U4 snRNA k-turn by single-molecule FRET measurements in the absence and presence of the 15.5K protein and its dependence on the $\mathrm{Na}^{+}$and $\mathrm{Mg}^{2+}$ ion concentration. We show that the unfolded U4 snRNA k-turn introduces a kink of $85^{\circ} \pm 15^{\circ}$ in an RNA double helix. While $\mathrm{Na}^{+}$and $\mathrm{Mg}^{2+}$ ions induce this more open conformation of the k-turn, binding of the $15.5 \mathrm{~K}$ protein was found to induce the tightly kinked conformation in the RNA that increases the kink to $52^{\circ} \pm 15^{\circ}$. By comparison of the measured FRET distances with a computer-modeled structure, we show that this strong kink is due to the $k$ turn motif adopting its folded conformation. Thus, in the free U4 snRNA, the k-turn exists only in an unfolded conformation, and its folding is induced by binding of the $15.5 \mathrm{~K}$ protein.
\end{abstract}

Keywords: U4 snRNA k-turn; 15.5K; protein-induced folding; multiparameter fluorescence detection; fluorescence resonance energy transfer; FRET

\section{INTRODUCTION}

In recent years, the number of resolved RNA structures has increased dramatically. This has made a systematic analysis and classification of RNA motifs possible (Klosterman et al. 2004; Tamura et al. 2004) and allowed us to extract general architectural principles (Leontis and Westhof 2003). The kink-turn (k-turn) motif is a recently identified structural element found in ribosomal and nonribosomal RNAs (Ban et al. 2000; Schluenzen et al. 2000; Vidovic et al. 2000; Klein et al. 2001). It comprises an internal loop that introduces a

\footnotetext{
${ }^{4}$ Present address: Program in Molecular Medicine, University of Massachusetts Medical School, Worcester, MA 01605, USA.

Reprint requests to: Filipp Oesterhelt, Heinrich-Heine-Universität Düsseldorf, Institut für molekulare Physikalische Chemie, Universitätsstraße 1, Geb. 26.32.02.44, 40225 Düsseldorf, Germany; e-mail: Filipp.oesterhelt@ uniduesseldorf.de; fax: 0211/81-12803.

Article and publication are at http://www.rnajournal.org/cgi/doi/ 10.1261/rna.2950605.
}

sharp kink into the helical axis. The general sequence features of the different k-turn variants are a canonical stem and a 3-nucleotide bulge usually followed by two noncanonical G-A base pairs. Almost all of the k-turns are found associated with proteins (Klein et al. 2001). Thus, the kturn motif is an important protein recognition element and an interesting candidate for protein-assisted RNA folding.

The U4 snRNA k-turn investigated here originates from the major spliceosome, a large ribonucleoprotein particle that catalytically removes introns from pre-messenger RNAs. It consists of smaller ribonucleoprotein particles that have to be assembled and disassembled for each splicing event. The spliceosomal U4 snRNA k-turn is located in the $5^{\prime}$-stem-loop of the U4 snRNA, offering a binding site for the $15.5 \mathrm{~K}$ protein (Nottrott et al. 1999). Binding of the $15.5 \mathrm{~K}$ protein to the $\mathrm{k}$-turn motif triggers the assembly of the U4/U6 ribonucleoprotein particle, since its recruitment is required for the subsequent binding of the $61 \mathrm{~K}$ and the 20/60/90K proteins (Nottrott et al. 2002). 
The structure of the complex formed by the $15.5 \mathrm{~K}$ protein and the $5^{\prime}$-stem-loop is known from a crystal structure analysis (Vidovic et al. 2000). However, as the X-ray structure was determined for the complex, there is no information about the conformation of the unbound RNA. Furthermore, little is known about the folding mechanism of the RNA, which may vary within the family of k-turn motifs. Recently, the unfolding of the U4 snRNA k-turn has been investigated by MD simulations (Cojocaru et al. 2005). There, it was shown that the flexibility of specific bases might be involved in the process of protein binding. However, these simulations were limited to 10 -nsec trajectories and did not involve ion binding. Experimental data are thus still needed to characterize the RNA's structural properties in solution and the dynamic changes of the RNA structure on longer time scales.

In order to detect conformational changes, the variation of intramolecular distances can be measured by Fluorescence Resonance Energy Transfer (FRET). The efficiency of energy transfer from a donor to an acceptor fluorophore is sensitive in the nanometer range and can be applied to single molecules. This makes it possible to detect structural heterogeneities and individual folding pathways of the investigated sample molecules. Single-molecule FRET measurements on several RNA structures have demonstrated the potential of this technique (Ha et al. 1999; Kim et al. 2002; Zhuang et al. 2002; Tan et al. 2003; Xie et al. 2004).

In principle, FRET measurements allow the calculation of intramolecular distances with a resolution of a few angstroms. However, sources of errors like orientational confinement or quenching of the fluorophores due to interaction with the RNA usually cannot be controlled by measuring either intensities or lifetimes, only. In contrast, the method of Multiparameter Fluorescence Detection (MFD) measures simultaneously all fluorescence parameters available from a single fluorophore (Eggeling et al. 2001; Margittai et al. 2003; Rothwell et al. 2003; Diez et al. 2004). This leads to a strong reduction of sources of errors in distance measurements compared to other techniques. The method of MFD is based on a confocal setup, in which the molecules diffuse through the focus and can be measured individually, allowing the analysis of mixtures of RNA and RNA-protein complexes.

Here, we applied this single-molecule MFD-FRET technique to investigate the interaction of the U4 snRNA k-turn and the $15.5 \mathrm{~K}$ protein to reveal conformational changes in the RNA structure.

\section{RESULTS}

\section{Design of sample molecules for U4 snRNA-15.5K interaction measurements by single-molecule FRET}

Folding of small RNA domains often takes place at subnanometer dimensions, but it may induce rearrangements of adjacent regions. This can amplify the small distance changes, which makes them amenable for FRET measurements. In order to detect conformational changes in a small RNA folding motif, it can be elongated artificially by attaching double-helical arms. By further attaching a donor and an acceptor to the ends of these arms, changes in the RNA structure can be measured directly by FRET. Thus, to analyze the folding of the k-turn motif in the U4 snRNA (Fig. 1A), we elongated this sequence by double-helical regions on both sides. The U4 snRNA $5^{\prime}$-stem-loop on one side of the k-turn motif was replaced by a double helix composed of six canonical base pairs, and the helical stem at the other side was elongated by two Watson-Crick base pairs to reduce helixopening fluctuations (Fig. 1A).

It is known that the interaction of the fluorophores with the bases at the end of a helical stem can induce quenching. To reduce this interaction, donor and acceptor were bound to the RNA by using an internal amino modification three bases away from the $3^{\prime}$-end. The length of the helical arms was chosen such that the fluorophores could neither disturb the folding of the k-turn sequence nor be disturbed by the binding of the $15.5 \mathrm{~K}$ protein.

To test the influence of the bulged A located close to the k-turn motif, also a modified binding motif was investigated in which the bulged A was removed and the $\mathrm{G} \cdot \mathrm{U}$ bases were replaced by a G-C Watson-Crick base pair. In addition, an RNA duplex was analyzed for reference, in which the k-turn motif was eliminated by removing or replacing all bases in the strand that usually contains the bulged nucleotides, thereby creating a double helix with only Watson-Crick base-pairings.

\section{MFD-FRET measurements on fluorescently labeled k-turn motif RNA probes}

The energy transfer from the donor to the acceptor is observed as a reduction in fluorescence intensity $F_{\mathrm{D}}$ of the donor and an increased intensity $F_{\mathrm{A}}$ of the acceptor. At the same time, the donor fluorescence lifetime is reduced owing to the energy transfer. Therefore, the transfer efficiency $E_{\mathrm{T}}$ can also be calculated from the donor fluorescence lifetime:

$$
E_{T}=\left[1+\frac{F_{D}}{F_{A}} \frac{\Phi_{F A}}{\Phi_{F D}}\right]^{-1}=1-\frac{\tau_{D(A)}}{\tau_{D(0)}}
$$

Here $\Phi_{\mathrm{FA}}$ and $\Phi_{\mathrm{FD}}$ are the acceptor and donor fluorescence quantum yields, respectively, and $\tau_{\mathrm{D}(\mathrm{A})}$ and $\tau_{\mathrm{D}(0)}$ the donor fluorescence lifetime in the presence and absence of the acceptor, respectively. The method of multiparameter fluorescence detection makes it possible to measure the two independent parameters, intensity and lifetime, simultaneously for each individual molecule. Thus, on each molecule two independent but simultaneous experiments are performed to measure the FRET efficiency, which allows one to detect and to eliminate many sources of errors.

From the ratio $F_{\mathrm{D}} / F_{\mathrm{A}}$ the distance $R_{\mathrm{DA}}$ between donor and acceptor can be calculated directly using Förster's for- 

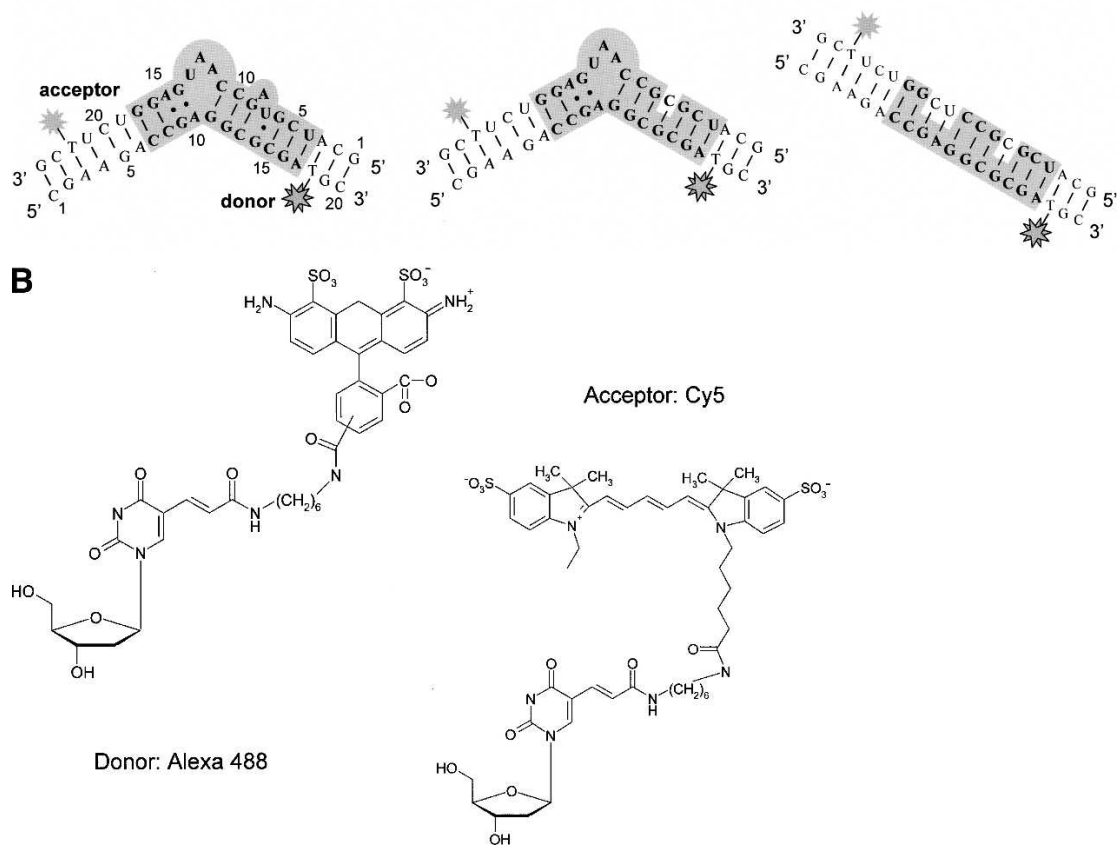

FIGURE 1. Sample sequences and used fluorophores. (A) To observe the conformational change of the U4 snRNA k-turn by FRET, the native U4 snRNA $5^{\prime}$-stem-loop sequence as indicated by the gray-shaded areas was elongated by double-helical segments at both ends. In addition to the native U4 snRNA k-turn sequence, a modified sequence missing the bulged $\mathrm{A}$ and the $\mathrm{G} \cdot \mathrm{U}$ mismatch was investigated. A linear A-RNA lacking the k-turn motif was used as a control RNA. (B) In all experiments, Alexa Fluor 488 carboxylic acid (Molecular Probes) and Cy5 (Amersham Pharmacia), both as succinimidyl ester activated derivatives, were used as donor and acceptor, respectively. These moieties were bound to the amino group of a 5-C6amino- $2^{\prime}$-deoxythymidine that was introduced into all four strands during synthesis. Both dyes are negatively charged, leading to repulsion from the RNA backbone and minimizing RNAfluorophore interactions.

mula $E_{\mathrm{T}}=1 /\left(1+\left(R_{\mathrm{DA}} / R_{0}\right)^{6}\right)$, with the Förster distance $R_{0}=0.211\left(J \kappa^{2} \Phi_{\mathrm{FD}} n^{-4}\right)^{1 / 6} \AA$. Here $J$ denotes the spectral overlap integral in $\mathrm{M}^{-1} \mathrm{~cm}^{-1} \mathrm{~nm}^{4}, \kappa$ the orientation factor, and $n$ the refraction index. According to Rothwell et al. (2003), we defined the reduced Förster distance $R_{0 \mathrm{r}}=R_{0} /$ $\Phi_{\mathrm{FD}}^{1 / 6}$, which is independent of the donor fluorescence quantum yield.

$$
R_{D A}=R_{0 r}\left[\frac{F_{D}}{F_{A}} \Phi_{F A}\right]^{1 / 6}
$$

The error has been estimated from the half-width of the distance distribution.

After calculating the distance from the intensity ratio $F_{\mathrm{D}} /$ $F_{\mathrm{A}}$ and evaluating the donor fluorescence lifetime $\tau_{\mathrm{D}}$ for each detected single molecule, the data are displayed as twodimensional (2D) histograms of all measured single-molecule events with the distance along the vertical axis and the donor lifetime along the horizontal axis (Fig. 2). The frequency of the bursts is indicated by a color code. If the distance and the donor lifetime are changed because of the Förster energy transfer only, all single-molecule events will be located along the curve describing the dependency between the distance and the donor lifetime (eq. 3).

$$
R_{D A}=R_{0 r}\left(\frac{\tau_{D(0)} k_{F D} \tau_{D(A)}}{\tau_{D(0)}-\tau_{D(A)}}\right)^{1 / 6}
$$

Here $k_{\mathrm{FD}}$ is the rate constant of the donor fluorescence emission. Any artifacts leading to a quenching of the donor will become visible as a deviation from this line in a horizontal direction, since the measured distance is independent of the donor fluorescence quantum yield (eq. 2). On the other hand, a quenching of the acceptor only affects the calculated distance $R_{\mathrm{DA}}$ and, thus, would become visible as a deviation in vertical direction. A simultaneous change of donor and acceptor fluorescence quantum yields can be excluded from the simultaneous measurement of those molecules that carry only the donor fluorophore. Therefore, all FRET efficiencies measured for molecules located along the curve given by equation 3 may be used to calculate correct distances.

Figure 2A-C shows the results for the three RNA duplexes containing the kturn motif, the modified motif where the bulged A and the G. U base pair were replaced by G$\mathrm{C}$, and the reference RNA without k-turn motif, respectively. In all three experiments, two species are visible.

After calculating the distances from the measured green and red signals, single-stranded RNAs carrying the donor only or duplexes in which the acceptor is bleached appear at distances above $90 \AA$ because of the background signal in the red channels originating from detector dark counts, cross-talk, and scattered light. Accordingly, these values represent no real distances. Owing to background subtraction, only single-molecule events with a red signal above the average red channel background are visible. The species with the short inter-dye distance represent the double strands showing FRET.

The comparison of Figure 2, A and B, shows that the distance in the FRET species does not change upon deletion of the bulged $A$ and the replacement of the $\mathrm{G} \cdot \mathrm{U}$ pair by GC. From the intensity ratio, a FRET efficiency of 0.37 for both, the native and the modified binding motifs, was calculated. Using a reduced Förster distance of $5.37 \mathrm{~nm}$, this corresponds to a distance of $5.6 \pm 0.3 \mathrm{~nm}$. In Figure 2B 

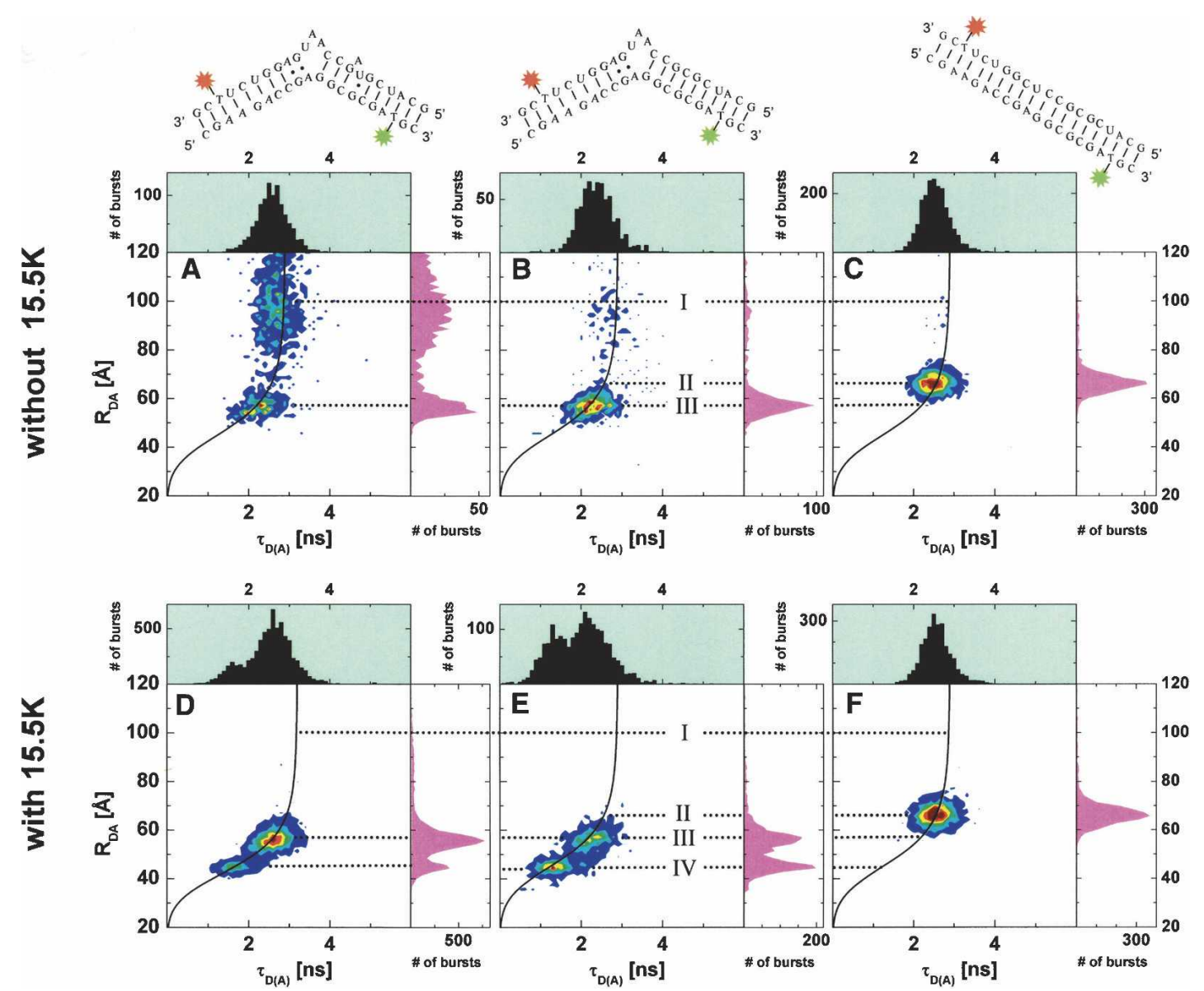

FIGURE 2. Multiparameter fluorescence measurements on the k-turn motif. $(A-C)$ The $2 \mathrm{D}$ histograms of the single-molecule events measured by Multiparameter Fluorescence Detection (MFD) for the RNA samples. The increasing number of molecules is shown by a color gradient. The species with the big inter-dye distance represent the molecules containing the donor only. The black sigmoidal line depicts the simultaneous change of distance and donor lifetime for all possible FRET efficiencies according to equation 3 with $\tau_{\mathrm{D}(0)}=2.9 \mathrm{nsec}, k_{\mathrm{FD}}=0.16 \mathrm{nsec}{ }^{-1}$. The horizontal line I indicates the donor-only population, while II and III refer to the reference duplex and the duplex including the native binding motif, respectively. The distances were calculated assuming a red fluorescence quantum yield of $\Phi_{\mathrm{FA}}=0.52$ and a reduced Förster distance $R_{0 \mathrm{r}}$ of $5.37 \mathrm{~nm} . R_{0 \mathrm{r}}$ was calculated assuming a refraction index $n=1.33$ and an orientation factor $\kappa^{2}=2 / 3$. The assumption of $\kappa^{2}=2 / 3$ was justified by the measured low donor fluorophore anisotropies of $0.061,0.068$, and 0.108 for the reference RNA, the duplex containing the native $\mathrm{k}$-turn and the RNA/protein complex, respectively (see Supplementary information at http://www.mpc.uni-duesseldorf.de/nanoanalytik/publications.htm). The acceptor anisotropies were measured only by FRET-mediated excitation. But since the linker used for the acceptor fluorophore is even longer than the one for the donor, we may assume the dynamic averaging condition to be fulfilled. The measurements were conducted in buffer A+ at an RNA concentration of $\sim 0.1 \mathrm{nM}$. $(D-F)$ The 2D histograms for the samples that additionally contain the $15.5 \mathrm{~K}$ protein at a concentration of $220 \mathrm{nM}$. A new species highlighted by line IV occurs in the two samples containing the k-turn motif, indicating that the RNA folds into a more compact structure upon protein binding. For the complex formation, buffer B was used. The measurements were done in buffer A+ at $0.1 \mathrm{nM}$ RNA concentration.

the species carrying only the donor fluorophore is depleted, while the relative number of molecules showing FRET is increased. This difference is due to the higher hybridization efficiency caused by the higher number of Watson-Crick base-pairings in the sample containing the modified k-turn motif. The reference sample without the k-turn motif, that contains only Watson-Crick base pairings, has the highest hybridization efficiency and accordingly shows almost no free single strands carrying the donor only (Fig. 2C).

The reference duplex in Figure $2 \mathrm{C}$ clearly shows a greater distance compared with the native and the modified k-turn motifs. These completely double-helical molecules have a lower FRET efficiency and, thus, a greater distance between donor and acceptor than the samples containing the k-turn motif. For these molecules, a FRET efficiency of 0.19 was calculated, corresponding to a donor-acceptor distance of $6.5 \pm 0.25 \mathrm{~nm}$. Thus, the FRET measurements clearly indicate that the bulged nucleotides of the k-turn motif induce a kink in the RNA helix. A contribution of the bulge induced by the additional $A$ and the $G \cdot \mathrm{U}$ base pair to the kink could not be observed. Assuming the RNA to kink in the phosphate backbone of the donor strand between G9 and A10, the angle that is formed by the kink can be roughly estimated from the one measured distance as 
$76^{\circ} \pm 15^{\circ}$ (see also Materials and Methods). A more precise calculation would require the measurement of several distances between the helical arms.

\section{Binding of the $15.5 \mathrm{~K}$ protein to the k-turn motif induces a strong kink in the RNA}

Figure 2D-F shows the same samples upon addition of the $15.5 \mathrm{~K}$ protein. In all samples the relative numbers of the molecules carrying the donor only are almost depleted. Accordingly, the number of double strands showing FRET transfer is increased. In the samples containing the native (Fig. 2A) and the modified k-turn motifs (Fig. 2B), a new species appears with a much higher FRET efficiency and, therefore, a much smaller inter-dye distance. In the reference sample containing no k-turn motif, no change is visible upon addition of the $15.5 \mathrm{~K}$ protein. This demonstrates that the new species represents the complex formed by specific binding of the $15.5 \mathrm{~K}$ protein to the k-turn motif. The high FRET efficiency shows that protein binding strongly enhances the kink in the RNA. From the measured distance of $4.5 \pm 0.2$ $\mathrm{nm}$, an angle of $48^{\circ} \pm 15^{\circ}$ can be roughly estimated. The result, that no high FRET species is visible before addition of the $15.5 \mathrm{~K}$ protein, indicates that binding of the $15.5 \mathrm{~K}$ protein is required for the folding of the k-turn motif.

Note that the shift of the species indicating the free duplex RNA is not complete because the experiments were done close to equilibrium conditions. The equilibrium constant for the U4 snRNA/15.5K complex formation was given by Watkins et al. (2000) to be $20 \mathrm{nM}$. It is beyond the scope of this investigation to determine the equilibrium constant from the numbers of bursts in the two species indicating the RNA duplex and the RNA-protein complex, which is not possible because of the uncertainty in the effective sample concentrations caused by loss of sample due to surface adsorption.

\section{Influence of salt on RNA structure and FRET efficiencies}

Previous studies have shown that the folding of many RNA structures is ion-dependent. Ions can bind specifically to RNA junctions and induce folding, or influence the RNA structure through unspecific electrostatic interaction (Auffinger et al. 2000, 2004; Kim et al. 2002). Here, the FRET efficiencies for the RNA molecules were measured in $90 \mathrm{mM}$ Tris-borate buffer with $\mathrm{Mg}^{2+}$ and $\mathrm{Na}^{+}$concentrations varying from 0 to $5 \mathrm{mM}$ and 0 to $200 \mathrm{mM}$, respectively.

The distances measured for all sample molecules show a saltdependent decrease with increasing concentration (Fig. 3). The sample molecules containing the U4 snRNA k-turn motif show a conformational transition that is induced by $\mathrm{Na}^{+}$ions at a concentration between 20 and $100 \mathrm{mM}$, while the $\mathrm{Mg}^{2+}$ ions induce a conformational transition that occurs already at a concentration below $0.1 \mathrm{mM}$. The influence of the $\mathrm{Mg}^{2+}$ and $\mathrm{Na}^{+}$ions on the RNA is not independent of each other. The combination of $\mathrm{Mg}^{2+}$ and $\mathrm{Na}^{+}$as used in the measurements for protein association again introduces a slight shortening of the measured distances (see Fig. 2; Table 1). This measured salt dependency shows that the unfolded U4 snRNA k-turn motif
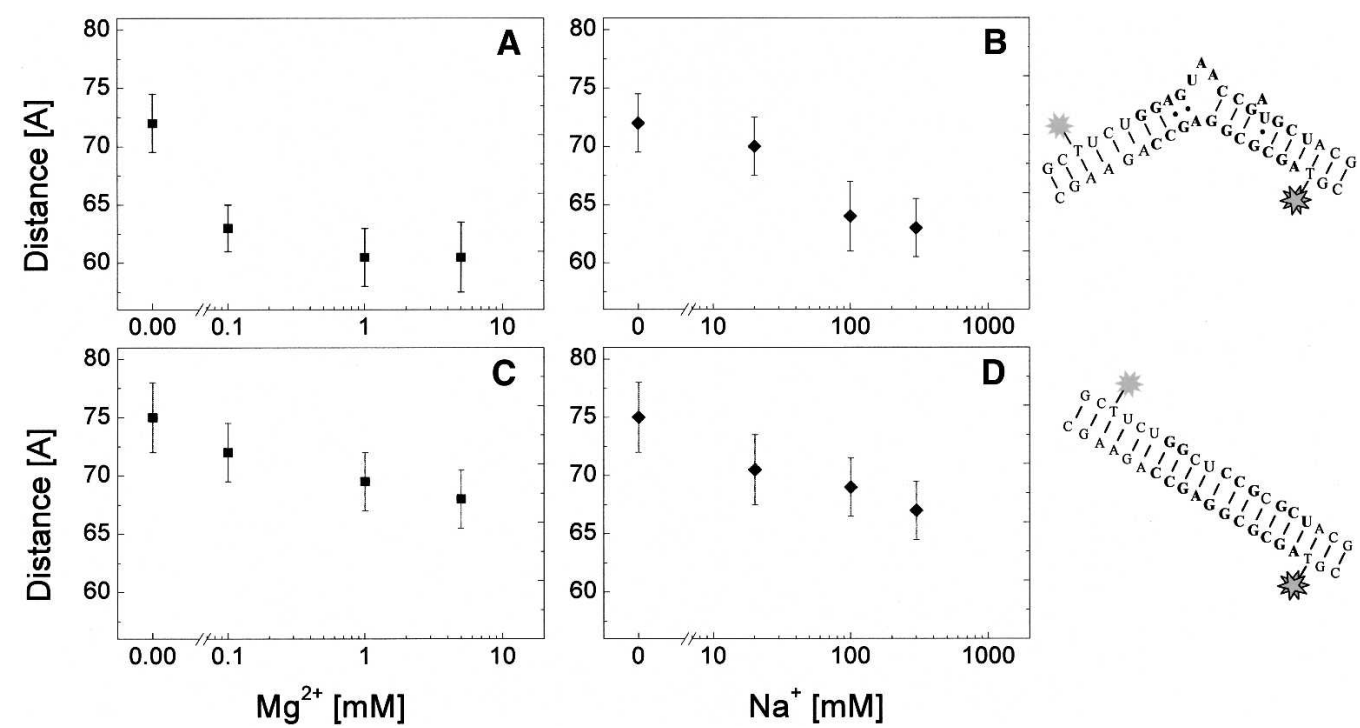

FIGURE 3. Salt dependence of the measured FRET efficiencies. $(A, B)$ Measured intensity ratios and the resulting calculated distances change only slightly with the salt concentration. This variation is much smaller than the one induced by binding of the $15.5 \mathrm{~K}$ protein. It is interesting to note that also the efficiency measured for the reference RNA slightly decreases with increasing salt concentration, indicating that the variation of the efficiency measured in the samples containing the k-turn is only partly due to a change in the RNA kink $(C, D)$. The salt dependence of the RNA structure was determined in buffer $\mathrm{C}$ with additional $\mathrm{NaCl}$ and $\mathrm{MgCl}_{2}$ as indicated in the respective graph. For the measurements without $\mathrm{Na}^{+}$and $\mathrm{Mg}^{2+}$ ions, EDTA at a concentration of $2 \mathrm{mM}$ was added. The concentration of the donor strand was $\sim 0.1 \mathrm{nM}$ during the measurements. 
TABLE 1. Distances and interhelical angles for different binding situations

\begin{tabular}{|c|c|c|c|c|}
\hline & \multicolumn{2}{|c|}{ FRET-measurements } & \multicolumn{2}{|c|}{ Modeled structure } \\
\hline & Distance & $\begin{array}{l}\text { Interhelical } \\
\text { angle }\end{array}$ & Distance & $\begin{array}{l}\text { Interhelical } \\
\text { angle }\end{array}$ \\
\hline No binding site & $6.5 \pm 0.25 \mathrm{~nm}$ & $180^{\circ}$ & $6.0 \pm 0.95 \mathrm{~nm}^{\mathrm{a}}$ & $180^{\circ \mathrm{a}}$ \\
\hline $\begin{array}{l}\text { Native binding motif } \\
\text { Native binding }\end{array}$ & $5.6 \pm 0.3 \mathrm{~nm}$ & $76 \pm 15^{\circ}$ & ---- & ---- \\
\hline motif $+15.5 \mathrm{~K}$ protein & $4.5 \pm 0.2 \mathrm{~nm}$ & $48 \pm 15^{\circ}$ & $4.2 \pm 1.1 \mathrm{~nm}^{\mathrm{b}}$ & $53^{o b}$ \\
\hline \multicolumn{5}{|l|}{ aA-RNA } \\
\hline \multicolumn{5}{|l|}{${ }^{\mathrm{b}}$ Crystal structure } \\
\hline \multicolumn{5}{|c|}{$\begin{array}{l}\text { For both the linear RNA and the k-turn RNA/15.5K complex, good agreement was found for } \\
\text { the measured and modeled distances. The free k-turn motif adopts an unfolded conforma- } \\
\text { tion that is clearly less kinked than the conformation found in the U4 snRNA k-turn/15.5K } \\
\text { complex. The interhelical angles were calculated based on the FRET measured distances, } \\
\text { assuming the helix to kink in the phosphate backbone of the donor strand between bases G9 } \\
\text { and A10. These distances were measured in buffer A+. }\end{array}$} \\
\hline
\end{tabular}

adopts a structure that is stabilized by the binding of $\mathrm{Na}^{+}$and $\mathrm{Mg}^{2+}$ ions.

However, our measurements clearly show that the ions cannot introduce the tightly kinked conformation that is induced by the $15.5 \mathrm{~K}$ protein. In the crystal structure of the U4 snRNA k-turn/15.5K complex, no localized $\mathrm{Mg}^{2+}$ ions were found (Vidovic et al. 2000), also suggesting that the ions may stabilize the k-turn loop in a more open conformation, but do not participate in the stabilization of the folded conformation.

\section{Comparison of distances obtained by FRET measurements and from molecular modeling}

To test the quality of our multiparameter FRET distance measurements, we modeled the RNA molecules under investigation together with the fluorophores. For the reference RNA without a k-turn motif, the aminoallyl-modified thymidine including the attached linkers and fluorophores was modeled into a straight A-RNA helix.

Since the FRET efficiency depends on the relative orientation of donor and acceptor, it is important that the fluorophores have a high rotational freedom, to allow rapid averaging of all orientations. Therefore, we used linkers of $\sim 6 \AA$ in length for Alexa 488 and $12 \AA$ for Cy5 (Fig. 1B).

However, this leads to an uncertainty of the fluorophores' positions. To quantify this uncertainty and to estimate the fluorophore positions, we performed MD simulations of the dyes attached to the RNA. A strong interaction of the fluorophores with the RNA in the experiment can be excluded on the basis of the simultaneously measured anisotropies for the donor and the acceptor fluorophores. For the anisotropy of the U4 snRNA k-turn/ $15.5 \mathrm{~K}$ complex, we measured 0.108 , and for the duplex containing the native binding motif, we measured an anisotropy of 0.068 . For the reference duplex, we got an anisotropy of 0.061 . All values were in good agreement with an overall rotational correlation time of $0.45 \mathrm{nsec}$ (see at http://www. mpc.uni-duesseldorf.de/nanoanalytik/ publications.htm).

Additionally, a strong interaction of the acceptor with the RNA would introduce a quenching of that fluorophore, which is not seen. Such acceptor quenching would be clearly visible as a deviation in the vertical $\left(R_{\mathrm{DA}}\right)$ direction of the sigmoidal curve in Figure 2, which depicts the simultaneous change of the distance and donor lifetimes due to a variation only in FRET efficiency. Thus, our experimental data imply that donor and acceptor do not interact strongly with the RNA. Therefore, the flexibility of the dyes should be mainly restricted by steric interactions. Water molecules bound to the RNA or electrostatic repulsion between the RNA and the fluorophore will lead to a further restriction of the possible fluorophore positions.

An upper limit of the dye flexibility is obtained by carrying out simple high-temperature vacuum simulations, neglecting electrostatic interactions. The RNA conformation was retained by harmonic constraints. This allows an extensive sampling of the sterically allowed configurational space of the dye. The obtained dye positions and orientations represent the largest ensemble obeying the steric restrictions of the dyes because of the RNA and the linkers. Note that the dynamics of the dyes are not investigated here, which, owing to the missing solvent and the high temperature, are not described correctly. From the ensembles of fluorophore positions obtained by the MD simulation, we calculated the mean position for the donor and the acceptor dye. For comparison with the measured distances, we took the distance between the donor and the acceptor fluorophores' mean positions (Fig. 4; Table 1). The respective errors are derived from the standard deviation also obtained from the MD ensemble. In the modeled straight A-RNA, the distance between the mean positions of donor and acceptor was calculated to be $6.0 \pm 0.95 \mathrm{~nm}$, in good agreement with the distance of $6.5 \pm 0.25 \mathrm{~nm}$ obtained experimentally by MFD-FRET.

Our FRET measurements indicate that the binding of the $15.5 \mathrm{~K}$ protein to the k-turn motif induces a strong kink in the RNA. Vidovic et al. (2000) resolved the structure of the crystallized $5^{\prime}$-stem-loop/15.5K complex by X-ray diffraction, where the RNA in the crystal was also found to adopt the strongly kinked conformation typical for the k-turn family (Klein et al. 2001). To compare the structure of the U4 snRNA k-turn/15.5K complex in solution with the $\mathrm{k}$ turn structure found in the crystal, we modeled the structure of our sample RNA based on the k-turn motif from the $\mathrm{X}$-ray structure. Thus, a structure was obtained where the 

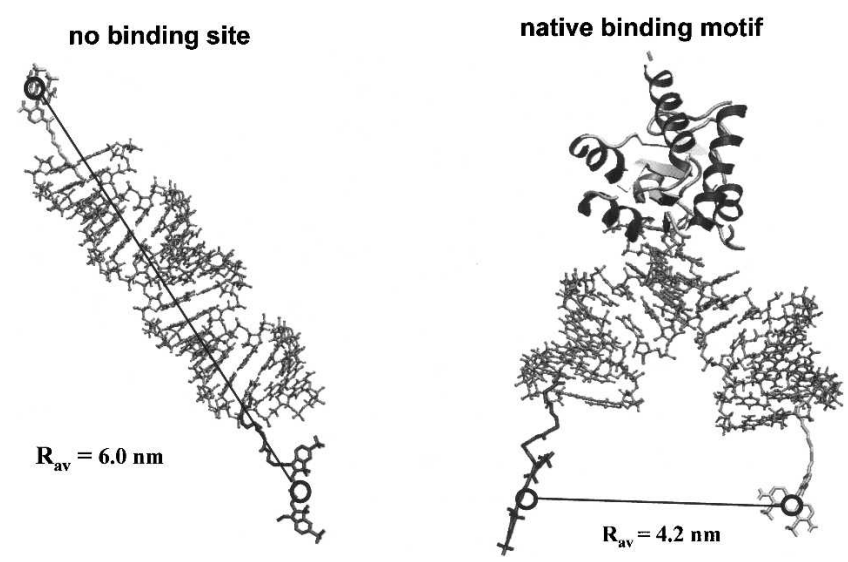

FIGURE 4. Modeling of the RNA and the fluorophores. To compare the measured distances with model structures, the positions of the fluorophores have to be considered. To account for the positional uncertainty introduced by the length of the linkers, a set of 10,000 possible positions of the fluorophores was calculated by moleculardynamic simulation, taking only steric hindrance of the RNA into account. To obtain an estimate of the fluorophores' positions and to compare the modeled structures with the measured distances, the average position for each fluorophore was calculated. The structure of the RNA-protein complex was modeled by elongating the crystal structure of the U4 snRNA k-turn with helical arms.

k-turn motif forms a strong kink between two helical arms carrying the fluorophores at their ends. To determine the fluorophore positions, we again calculated their mean position from vacuum MD simulations that took into account only steric hindrance. The obtained distance between the mean positions of donor and acceptor is $4.2 \pm 1.1 \mathrm{~nm}$. This agrees well with the distance of $4.5 \pm 0.2 \mathrm{~nm}$ measured by MFD-FRET in the RNA/15.5K complex. This indicates that in free solution the k-turn motif in the U4 snRNA k-turn/ $15.5 \mathrm{~K}$ complex adopts a folded conformation similar to the one found by X-ray crystallography. In addition, the folded structure is not adopted in the absence of the $15.5 \mathrm{~K}$ protein. Thus, we conclude that the folding of the k-turn motif is induced by binding of the $15.5 \mathrm{~K}$ protein.

\section{DISCUSSION}

The experiments described above demonstrate the potential of single molecule MFD-FRET as a quantitative tool for intramolecular distance measurements. The measurement of single molecules diffusing through the detection focus of a confocal setup allows us to measure several independent fluorescence parameters simultaneously. This makes it possible to exclude quenching of sample-fluorophore interactions, which would introduce errors in the distance calculation. At the same time, different conformational species can be separated in complex samples, and distances for all species can be calculated from one measurement.

Our measurements on the U4 snRNA k-turn/15.5K mixture show two species in the 2D histograms, representing the RNA duplex and the RNA/15.5K complex, respectively, indicating that the U4 snRNA k-turn has only two preferred conformations, an unfolded and a folded one. The fact that two separate species are observed shows that the molecules must stay in the folded and unfolded conformations much longer than the experimental observation time of several milliseconds, which is given by the diffusion of the molecules through the confocal detection volume. Otherwise a significant fraction of the detected single-molecule events would show a switch from one conformation to the other, leading to an averaging of the two conformations and a wrong calculation of distances. Thus, the clear separation of the two species shows that the transition events are rare and the transition occurs on a short time scale. To confirm this, we analyzed time traces of the individual events, but could not detect any switching between different conformations at times down to $1 \mathrm{msec}$ (data not shown). Here it is also important to point out, that neither could short-lived folded conformations be observed in the individual time traces, either in the presence or in the absence of the $15.5 \mathrm{~K}$ protein. In concordance with this, Cojocaru et al. (2005) showed in a molecular-dynamic simulation that in the absence of the $15.5 \mathrm{~K}$ protein, the k-turn motif unfolds partially already after $10 \mathrm{nsec}$.

To detect possible faster folding-unfolding events, we performed a species-selective fluorescence correlation analysis (FCS) (Margittai et al. 2003; Felekyan et al. 2005). Therefore, we gathered all photon data for the k-turn RNA without $15.5 \mathrm{~K}$ protein and performed a cross-correlation analysis between the measured red and green intensities. There, down to the $10-\mu \mathrm{sec}$ time scale, no anticorrelation was observed, which would indicate a fluctuation in the donor-acceptor distance (data not shown). Moreover, strong fluctuations, which would be detectable at times down to $100 \mathrm{nsec}$, were not observed. This shows that the species indicating the unfolded conformation is truly one species and does not result from averaging of different long-lived conformations. In addition, the absence of long-lived folded conformations shows that the complex of the U4 snRNA k-turn and the $15.5 \mathrm{~K}$ protein must be formed either by an induced-fit process or by conformational capture of a short-lived folded conformation.

The folding of RNA structures is often dependent on the interaction with ions. Our measurements on the U4 snRNA kturn motif revealed a conformational transition that can be induced by $\mathrm{Na}^{+}$and $\mathrm{Mg}^{2+}$ ions at a concentration between 20 and $100 \mathrm{mM}$, and below $0.1 \mathrm{mM}$, respectively (Fig. 3A,B). A similar result was found by Goody et al. (2004) for the KT-7 kturn motif from helix 7 of the 23S rRNA by frequency-domain lifetime measurements. This indicates that the unfolded U4 snRNA k-turn motif adopts a more open conformation, which is stabilized by the binding of $\mathrm{Na}^{+}$and $\mathrm{Mg}^{2+}$ ions. But, in contrast to the KT-7 k-turn motif, ion binding is not sufficient to introduce the tightly kinked folded conformation, which is only induced by the $15.5 \mathrm{~K}$ protein. 
Since we analyze single molecules, new long-lived (several milliseconds) conformations that might be induced by ions binding to the k-turn motif would appear as new species in the $2 \mathrm{D}$ histograms, which is not observed. Also, the analysis of the individual time traces and a species-selective correlation analysis at different salt concentrations could not reveal a switching between different conformations. This finding, and the high concentration of monovalent ions needed to induce the conformational change, suggest that the ions interact with the U4 snRNA k-turn by a "diffusely bound" mechanism, but do not bind specifically to the motif, nor do they induce a conformational switch to the folded structure.

However, the salt dependency of the measured FRET distances has to be interpreted carefully, since even the FRET efficiency measured for the double-helical reference RNA that did not contain any binding motif showed a weak dependency on the ion concentration (Fig. 3C,D). Such a shift of the distance could be explained by a structural change in the double-helical RNA, probably induced by electrostatic shielding or the binding of ions to the RNA backbone. But also a weak influence of the ions on the range of possible fluorophore positions cannot be excluded completely and could lead to a misinterpretation of the measured salt-dependent distances as a small conformational change in the RNA. The electrostatic shielding may be reflected in the superposing effect of the $\mathrm{Na}^{+}$and $\mathrm{Mg}^{2+}$ ions. Upon simultaneous adding of $\mathrm{Na}^{+}$and $\mathrm{Mg}^{2+}$ ions, the calculated distance is even shortened (Fig. 2).

Here it is interesting to note that with decreasing salt concentration, our single-molecule measurements show a depopulation of the species indicating the RNA duplex and an increase of the one indicating the single-stranded RNA in addition to the shift of the measured distance. In ensemble measurements that detect only intensities, a shift of the species and a change of their population could not be distinguished and would not allow calculating correct FRET efficiencies.

In the U4 snRNA-5'-stem-loop, the k-turn motif is flanked by two G-C Watson-Crick base pairs at both sides, followed by a UUUAU loop at one side and a double-helical stem including a bulged $A$ and a $G \cdot U$ base pair at the other side. The X-ray structure of the U4 snRNA- $5^{\prime}$ stem-loop/15.5K complex could not reveal any defined structure of the terminal loop. In our sample sequences, the loop was replaced by a double-helical strand. This is likely to induce a stabilization of the two G-C pairs and thus might stabilize the folded form of the k-turn motif. In contrast, we observed no folding of the k-turn motif in the absence of the $15.5 \mathrm{~K}$ protein. Thus, we may conclude that also the k-turn motif in the native U4 snRNA- $5^{\prime}$-stemloop is unfolded in the absence of the $15.5 \mathrm{~K}$ protein and folds only upon protein binding.

Comparison of the measured distances with the modeled structures including the probe dyes showed that the structure of the folded U4 snRNA k-turn in solution is very similar to the one found by X-ray crystallography. In absence of the $15.5 \mathrm{~K}$ protein, the k-turn is found to be unfolded and to form a significantly less kinked structure. From our measurements we may estimate the kink introduced into the RNA double helix by the unfolded k-turn motif to be $76^{\circ} \pm 15^{\circ}$.

\section{MATERIALS AND METHODS}

\section{Molecules}

The fluorescently labeled oligonucleotides (Fig. 1A,B) were synthesized commercially by IBA GmbH. To introduce an amino functionality for the attachment of the fluorophores, a 5-C6-amino- $2^{\prime}$ deoxythymidine was introduced into all strands during the oligonucleotide synthesis. Succinimidyl-ester-activated fluorophores were allowed to react with the oligonucleotides, followed by a PAGE purification to separate unbound fluorophores. As a donor, Alexa Fluor 488 carboxylic acid (Molecular Probes) was used, and as an acceptor Cy5 (Amersham Pharmacia) (Fig. 1B).

For hybridization of the two respective oligonucleotides, both RNA strands were combined in $50 \mathrm{mM}$ Tris- $\mathrm{HCl}, 150 \mathrm{mM} \mathrm{NaCl}, 1$ $\mathrm{mM}$ EDTA, and $5 \mathrm{mM} \mathrm{MgCl}_{2}$ at $\mathrm{pH} 7.6$ (buffer A) at a donoracceptor ratio of $1: 3$ and a donor strand concentration of $10 \mu \mathrm{M}$. The samples were heated to $80^{\circ} \mathrm{C}$ and cooled to room temperature overnight. For the single-molecule measurements, the samples were diluted to $\sim 0.1 \mathrm{nM}$ in buffer A plus $0.4 \mathrm{mM}$ vitamin $\mathrm{C}$ (buffer $\mathrm{A}+$ ) to prevent bleaching of the fluorophores. For experiments with protein, the hybridization buffer contained $20 \mathrm{mM}$ HEPES-KOH, $150 \mathrm{mM} \mathrm{KCl}, 1.5 \mathrm{mM} \mathrm{MgCl}_{2}, 0.2 \mathrm{mM}$ EDTA, and $0.1 \%(\mathrm{v} / \mathrm{v})$ Triton X-100 at $\mathrm{pH} 7.9$ (buffer B). For complex formation, the preformed RNA duplex $(0.05 \mu \mathrm{M})$ was heated together with the recombinant $15.5 \mathrm{~K}$ protein $(2 \mu \mathrm{M})$ and Escherichia coli tRNA $(0.5 \mathrm{~g} / \mathrm{L})$ to $36^{\circ} \mathrm{C}$, and the mixture was allowed to cool down to room temperature within $2 \mathrm{~h}$. The human $15.5 \mathrm{~K}$ protein was purified according to Nottrott et al. (1999). Again, the samples were diluted to $\sim 0.1 \mathrm{nM}$ in buffer $\mathrm{A}+$ for single-molecule experiments. In order to prevent dissociation of the RNA/15.5K complex due to dilution, $15.5 \mathrm{~K}$ protein was added to the measurements at a final concentration of $220 \mathrm{nM}$. For all measurements that included protein, the supporting glass surface was passivated with $0.1 \mathrm{~g} / \mathrm{L}$ tRNA for $15 \mathrm{~min}$ to reduce loss of sample due to adsorption to the surface.

The salt dependence of the RNA structure was determined in 90 $\mathrm{mM}$ Tris-borate buffer, $0.4 \mathrm{mM}$ vitamin $\mathrm{C}$ at $\mathrm{pH} 8.3$, with additional $\mathrm{NaCl}$ and $\mathrm{MgCl}_{2}$, respectively (buffer C). The respective final concentrations of $\mathrm{NaCl}$ and $\mathrm{MgCl}_{2}$ are given in Figure 3. For the measurements without $\mathrm{Na}^{+}$and $\mathrm{Mg}^{2+}$ ions, EDTA at a concentration of $2 \mathrm{mM}$ was added.

\section{SETUP}

The MFD setup was previously described in detail (Rothwell et al. 2003). In short, single-molecule FRET was performed using a confocal epi-illuminated microscope with excitation by a linearpolarized, active mode-locked $(73 \mathrm{MHz})$ argon-ion laser (Coherent) operated at $496 \mathrm{~nm}\left(\sim 50 \mathrm{~kW} / \mathrm{cm}^{2}\right)$, which was focused into solution by a $60 \times 1.2$ water immersion objective. The detection volume resulted in $2 \mathrm{fl}$, as estimated from fluorescence correlation 
spectroscopy (FCS). The diffusion time for the reference fluorophore Rh110 was 0.23 msec. The excitation light was separated from the fluorescence light by a 505 LP dichroic mirror, subsequently passing a $100-\mu \mathrm{m}$ pinhole for cutting out the scattered light. The fluorescence was consecutively separated into its parallel and perpendicular components (polarizing beamsplitter) as well as the green from the red photons (dichroic mirror 620DCLX or 595DCLP, band pass filters: HQ535/46, and HQ720/150, respectively; Chroma Technology Corp.). The photons were detected by four avalanche photodiodes (SPCM-AQR; Perkin Elmer Optoelectronics) coupled to a PC-BIFL card (SPC 130; Becker \& Hickl $\mathrm{GmbH}$ ) in the MFD-mode. For each photon, the arrival time after the laser pulse, the interphoton time since the last photon, the polarization, and the spectral range were recorded.

\section{Data treatment and analysis}

The MFD data registration and processing were also previously described in detail (Eggeling et al. 1998; Schaffer et al. 1999). The analysis was performed with Lab-View programs developed in our own laboratory. Fluorescence bursts resulting from molecules diffusing through the open confocal volume were distinguished from the background by applying the following criteria of a minimum of 160 consecutive photons with an interphoton time below $100 \mu \mathrm{sec}$ averaged over 30 subsequently registered photon counts. The photons of each burst were integrated in fluorescence decay histograms-one for each detector-describing photon arrival times relative to the incident laser pulse. From these histograms, fluorescence lifetime and anisotropy were deduced by fitting monoexponential decays with a convolution of the instrumental response function to each burst using a maximum likelihood estimator with linear regression. Furthermore, the count rate in the green and red detectors during the burst was determined. Both, the donor-to-acceptor fluorescence intensity ratio as well as the lifetime can be subsequently used to determine the energy transfer efficiency between the two fluorophores (eqs. 2 and 3). For this purpose, $F_{\mathrm{D}}$ and $F_{\mathrm{A}}$ are determined from the green and red signals by correcting them for the background counts, $B_{\mathrm{G}}$ and $B_{\mathrm{R}}$, detection efficiencies, $g_{\mathrm{G}}$ and $g_{\mathrm{R}}$, and spectral cross-talk, $\alpha$, according to equation 3 from Rothwell et al. (2003) (typical values are: $B_{\mathrm{G}}=2.9 \mathrm{kHz}, B_{\mathrm{R}}=0.9$ $\mathrm{kHz}, g_{\mathrm{G}}=0.36, g_{\mathrm{R}}=0.53$, and $\left.\alpha=0.019\right)$.

A detailed species-selective analysis of the fluorescence decay of donor-only molecules resulted in a double exponential fluorescence decay with the fluorescence lifetimes, $\tau_{\mathrm{DN}}$, and species amplitudes, $x_{\mathrm{DN}}\left(\tau_{\mathrm{D} 1}=3.35 \mathrm{nsec}, x_{\mathrm{D} 1}=0.78\right.$; and $\tau_{\mathrm{D} 2}=0.63 \mathrm{nsec}$, $x_{\mathrm{D} 2}=0.22$ ), indicating a more complex fluorescence quenching of the donor dye. The fit included an offset of $5.5 \%$ of the total fluorescence signal that originates from after-pulsing of the APDs. Quenching of the donor fluorophore does not perturb the FRET process as expressed by the "reduced Förster radius"; however, this competing process must be taken into account for the calculation of the appropriate species-weighted donor fluorescence lifetime. Therefore, a polynomial correction function was generated by simulating FRET to obtain the quadratic correction factors of $a=-0.0285, b=0.9401$, and $c=0.0121$. These values were used to rescale all experimentally determined lifetime values to their photophysically meaningful ones (Rothwell et al. 2003).

In addition, we corrected the data for the error introduced by the offset signal due to after-pulsing of the APDs. Since the fluorescence lifetime for each burst was calculated only by a mono-exponential fit without taking background into account, these values needed a further correction. Therefore, we fitted all the fluorescence decay data of the species donor only, RNA duplex, and U4 snRNA/15.5K complex, respectively, by a monoexponential decay, with and without taking into account the afterpulsing offset. The latter calculations yielded lifetimes on average $0.3 \mathrm{nsec}$ longer than the first ones, allowing us to correct the fluorescence lifetimes for offset by subtracting this value.

The interhelical angles were calculated based on the FRET-measured distances, assuming the helix to kink in the phosphate backbone of the donor strand between bases G9 and A10. We assumed the fluorophores to be located on the helical axis and calculated their distance from the kink by dividing the experimentally determined distance of $6.5 \mathrm{~nm}$ between donor and acceptor fluorophores on the linear reference RNA proportionately to the number of base pairs in each helical arm. In this calculation, we neglected the slight mean off-axis positioning of the fluorophores and a possible rotation of the helical arms. The error of the angle was estimated by recalculating the angle including a shortening/elongating of the inter-fluorophore distance by the mean fluorophore linker length as derived from the MD simulation.

\section{Molecular modeling}

Vacuum MD simulations of the two dyes attached to the RNA were carried out at elevated temperature $(T=2000 \mathrm{~K})$, using the GROMACS simulation software (Berendsen et al. 1995). In the simulations, the modeled RNA structure was restrained by strong harmonic potentials, while the dyes were free to move. The force field parameters for the dyes were adapted from analogous groups in the GROMACS force field. Since only steric hindrances were of interest here, electrostatic interactions were not included in the simulations. The simulation time was $10 \mathrm{nsec}$ in total. Coordinates were saved every 1 psec for further analysis, yielding an ensemble of 10,000 fluorophore configurations.

The model structures were assembled in VMD 1.8.2 (Humphrey et al. 1996).

\section{ACKNOWLEDGMENTS}

We thank Enno Schweinberger for fruitful discussions concerning fluorophore-nucleic acid interactions. We are grateful to Vova Kudryavtsev, Suren Felekyan, and Matthiew Antonik for programming Lab-View software that allows fast analysis of multiparameter fluorescence data. This work was supported by grants from the Deutsche Forschungsgemeinschaft (LU294/12-1), Fonds der Chemischen Industrie and Bundesministerium für Bildung, Forschung und Technologie (BioFuture Grant 0311865 and NanotechnologieNachsuchswettbewerb 03N8714).

Received April 27, 2005; accepted June 29, 2005.

\section{REFERENCES}

Auffinger, P. and Westhof, E. 2000. Water and ion binding around RNA and DNA (C, G) oligomers. J. Mol. Biol. 300: 1113-1131.

Auffinger, P., Bielecki, L., and Westhof, E. 2004. Anion binding to nucleic acids. Structure 12: $379-388$.

Ban, N., Nissen, P., Hansen, J., Moore, P.B., and Steitz, T.A. 2000. The complete atomic structure of the large ribosomal subunit at $2.4 \AA$ resolution. Science 289: 905-920. 
Berendsen, H.J.C., Spoel, D., and Drunen, R. 1995. GROMACS: A message-passing parallel molecular dynamics implementation. Comp. Phys. Comm. 91: 43-56.

Cojocaru, V., Nottrott, S., Klement, R., and Jovin, T.M. 2005. The snRNP $15.5 \mathrm{~K}$ protein folds its cognate K-turn RNA: A combined theoretical and biochemical study. RNA 11: 197-209.

Diez, M., Zimmermann, B., Börsch, M., König, M., Schweinberger, E., Steigmiller, S., Reuter, R., Felekyan, S., Kudryavtsev, V., Seidel, C.A.M., et al. 2004. Proton-powered subunit rotation in single membranebound $\mathrm{F}_{0} \mathrm{~F}_{1}$-ATP synthase. Nat. Struct. Mol. Biol. 11: 135-141.

Eggeling, C., Fries, J.R., Brand, L., Günther, R., and Seidel, C.A.M. 1998. Monitoring conformational dynamics of a single molecule by selective fluorescence spectroscopy. Proc. Natl. Acad. Sci. 95: 1556-1561.

Eggeling, C., Berger, S., Brand, L., Fries, J.R., Schaffer, J., Volkmer, A., and Seidel, C.A.M. 2001. Data registration and selective singlemolecule analysis using multi-parameter fluorescence detection. J. Biotechnol. 86: 163-180.

Felekyan, S., Kühnemuth, R., Kudryavtsev, V., Sandhagen, C., Becker, W., and Seidel, C.A.M. 2005. Full correlation from picoseconds to seconds by time-resolved and time-correlated single photon detection. Rev. Sci. Instr. (in press).

Goody, T.A., Melcher, S.E., Norman, D.G., and Lilley, D.M. 2004. The kink-turn motif in RNA is dimorphic, and metal ion-dependent. RNA 10: 254-264.

Ha, T., Zhuang, X.W., Kim, H.D., Orr, J.W., Williamson, J.R., and Chu, S. 1999. Ligand-induced conformational changes observed in single RNA molecules. Proc. Natl. Acad. Sci. 96: 9077-9082.

Humphrey, W., Dalke, A., and Schulten, K. 1996. VMD: Visual molecular dynamics. J. Mol. Graphics 14: 33-38.

Kim, H.D., Nienhaus, G.U., Ha, T., Orr, J.W., Williamson, J.R., and Chu, S. 2002. $\mathrm{Mg}^{2+}$-dependent conformational change of RNA studied by fluorescence correlation and FRET on immobilized single molecules. Proc. Natl. Acad. Sci. 99: 4284-4289.

Klein, D.J., Schmeing, T.M., Moore, P.B., and Steitz, T.A. 2001. The kinkturn: A new RNA secondary structure motif. EMBO J. 20: 4214-4221.

Klosterman, P.S., Hendrix, D.K., Tamura, M., Holbrook, S.R., and Brenner, S.E. 2004. Three-dimensional motifs from the SCOR, structural classification of RNA database: Extruded strands, base triples, tetraloops and U-turns. Nucleic Acids Res. 32: 2342-2352.

Leontis, N.B. and Westhof, E. 2003. Analysis of RNA motifs. Curr. Opin. Struct. Biol. 13: 300-308.

Margittai, M., Widengren, J., Schweinberger, E., Schröder, G.F., Felekyan, S., Haustein, E., König, M., Fasshauer, D., Grubmüller, H., Jahn, R., et al. 2003. Single-molecule fluorescence resonance energy transfer reveals a dynamic equilibrium between closed and open conformations of syntaxin 1. Proc. Natl. Acad. Sci. 100: 1551615521.

Nottrott, S., Hartmuth, K., Fabrizio, P., Urlaub, H., Vidovic, I., Ficner, R., and Lührmann, R. 1999. Functional interaction of a novel $15.5 \mathrm{~K}$ [U4/U6.U5] tri-snRNP protein with the $5^{\prime}$ stem-loop of U4 snRNA. EMBO J. 18: 6119-6133.

Nottrott, S., Urlaub, H., and Lührmann, R. 2002. Hierarchical, clustered protein interactions with U4/U6 snRNA: A biochemical role for U4/U6 proteins. EMBO J. 21: 5527-5538.

Rothwell, P.J., Berger, S., Kensch, O., Felekyan, S., Antonik, M., Wöhrl, B.M., Restle, T., Goody, R.S., and Seidel, C.A.M. 2003. Multi-parameter single-molecule fluorescence spectroscopy reveals heterogeneity of HIV-1 reverse transcriptase:primer/template complexes. Proc. Natl. Acad. Sci. 100: 1655-1660.

Schaffer, J., Volkmer, A., Eggeling, C., Subramaniam, V., Striker, G., and Seidel, C.A.M. 1999. Identification of single molecules in aqueous solution by time-resolved fluorescence anisotropy. $J$. Phys. Chem. A 103: 331-336.

Schluenzen, F., Tocilj, A., Zarivach, R., Harms, J., Gluehmann, M., Janell, D., Bashan, A., Bartels, H., Agmon, I., Franceschi, F., et al. 2000. Structure of functionally activated small ribosomal subunit at 3.3 A resolution. Cell 102: 615-623.

Tamura, M., Hendrix, D.K., Klosterman, P.S., Schimmelman, N.R., Brenner, S.E., and Holbrook, S.R. 2004. SCOR: Structural classification of RNA, version 2.0. Nucleic Acids Res. 32: D182-D184.

Tan, E., Wilson, T.J., Nahas, M.K., Clegg, R.M., Lilley, D.M., and Ha, T. 2003. A four-way junction accelerates hairpin ribozyme folding via a discrete intermediate. Proc. Natl. Acad. Sci. 100: 9308-9313.

Vidovic, I., Nottrott, S., Hartmuth, K., Lührmann, R., and Ficner, R. 2000. Crystal structure of the spliceosomal $15.5 \mathrm{~K}$ protein bound to a U4 snRNA fragment. Mol. Cell 6: 1331-1342.

Watkins, N.J., Ségault, V., Charpentier, B., Nottrott, S., Fabrizio, P., Bachi, A., Wilm, M., Rosbash, M., Branlant, C., and Lührmann, R 2000. A common core RNP structure shared between the small nucleoar box C/D RNPs and the spliceosomal U4 snRNP. Cell 103: 457-466.

Xie, Z., Srividya, N., Sosnick, T.R., Pan, T., and Scherer, N.F. 2004. Single-molecule studies highlight conformational heterogeneity in the early folding steps of a large ribozyme. Proc. Natl. Acad. Sci. 101: 534-539.

Zhuang, X., Kim, H., Pereira, M.J.B., Babcock, H.P., Walter, N.G., and $\mathrm{Chu}$, S. 2002. Correlating structural dynamics and function in single ribozyme molecules. Science 296: 1473-1476. 

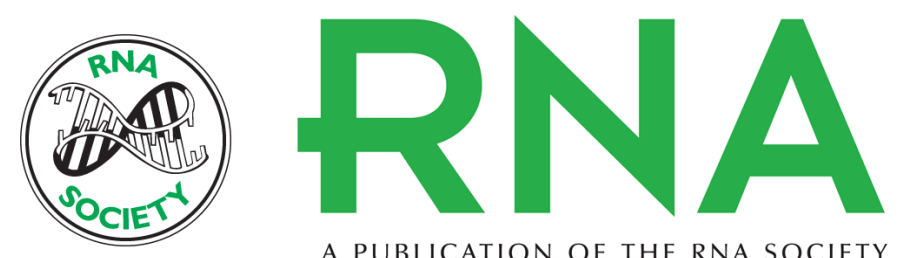

A PUBLICATION OF THE RNA SOCIETY

\section{Detecting protein-induced folding of the U4 snRNA kink-turn by single-molecule multiparameter FRET measurements}

ANNA K. WOZNIAK, STEPHANIE NOTTROTT, EVA KÜHN-HÖLSKEN, et al.

RNA 2005 11: 1545-1554

References This article cites 27 articles, 14 of which can be accessed free at:

http://rnajournal.cshlp.org/content/11/10/1545.full.html\#ref-list-1

License

Email Alerting Receive free email alerts when new articles cite this article - sign up in the box at the Service top right corner of the article or click here. 